\title{
VIOLENCIA OBSTÉTRICA: PERCEPCIÓN DE LAS USUARIAS SONORENSES
}

\author{
OBSTETRIC VIOLENCE: PERCEPTION OF WOMEN FROM SONORA.
}

\author{
OSMAR EFRÉN FIGUEROA PALOMINO* RUBÉN HURTADO LAGARDA** DIANA GUADALUPE VALENZUELA CORONADO** \\ JOSÉ DANIEL BERNAL CRUZ** CRISTIAN DAVID DUARTE GUTIERREZ** FABIOLA ARANTZA CAZARES GONZALEZ**
}

\begin{abstract}
Introduction: Pregnancy is a stage in which women are vulnerable, so care within hospital institutions during childbirth has a significant impact on their physical and mental health. Obstetric violence is one that is exercised within health institutions against pregnant women during childbirth care, and is still an important public health problem in Mexico.
\end{abstract}

Objective: The objective of the present study is to know the perception of the users of the health services about the existence of obstetric violence.

Methodology: Quantitative, descriptive study. The type of sampling was for convenience through the snowball technique, 45 participants were included. The data collection was through the Test to denounce obstetric violence in hospitals in Extremadura, adapted to the Mexican population, consists of 11 dichotomous questions.

Results: $37.7 \%$ of the surveyed women perceived some type of obstetric violence by health personnel. The order of not walking (51.1\%), criticism of crying or screaming (44.4\%), being unable to express fears or concerns $(37.8 \%)$, nicknames or diminutives $(28.9 \%)$, deny changes in position and deny contact with the newborn (26.7\%) and ironic comments, disqualifying, jokes (22.2\%).

Conclusions: Although the highest percentages are not inclined to affirmative in relation to actions considered as acts of obstetric violence, it is of great relevance to emphasize that these acts are present in considerable percentages.

Key words: Violence, obstetric delivery, patient care (DeCS, BIREME).

\section{RESUMEN:}

Ttroducción: El embarazo es una etapa en que la mujer se encuentra vulnerable, por lo que la atención dentro de las instituciones hospitalarias durante el parto tiene un impacto importante sobre su salud física y mental. La violencia obstétrica es aquella que se ejerce dentro de las instituciones de salud contra la mujer embarazada durante la atención del parto, y actualmente sigue siendo una problemática importante de salud pública en México.

Objetivo: El objetivo del presente estudio es conocer la percepción de las usuarias de los servicios de salud sobre la existencia de violencia obstétrica.

Metodología: Tipo de estudio, cuantitativo, descriptivo. El tipo de muestreo fue por conveniencia a través de la técnica de bola de nieve, se incluyeron 45 participantes. La recolección de datos fue mediante el Test para denunciar la violencia obstétrica en los hospitales extremeños, adaptado a población mexicana, consta de 11 preguntas dicotómicas.

Resultados: El 37.7\% de las mujeres encuestadas percibieron algún tipo de violencia obstétrica por parte del personal de salud. Con mayor frecuencia fue percibida la orden de no deambular (51.1\%), las críticas por llorar o gritar (44.4\%), imposibilidad para manifestar miedos o inquietudes $(37.8 \%)$, sobrenombres o diminutivos (28.9\%), impedir cambios de posición e impedir contacto con el recién nacido $(26.7 \%)$ y comentarios irónicos, descalificantes, chistes (22.2\%).

Conclusiones: Si bien, los porcentajes mayores no se inclinan por lo afirmativo en cuestión de acciones consideradas como actos de violencia obstétrica, es de gran relevancia destacar que dichos actos están presentes en porcentajes considerables.

Palabras clave: Violencia, parto obstétrico, atención al paciente (DeCS, BIREME).

*Estudiante de Licenciatura en Enfermería. Departamento de Enfermería. Universidad de Sonora. Correo para correspondencia: fepopalomino@ gmail.com
** Estudiante de Licenciatura en Enfermería. Departamento de Enfermería. Universidad de Sonora. 


\section{INTRODUCCIÓN}

A nivel mundial la atención a la salud materna es una prioridad que determina la calidad del sistema de salud de cada nación. Muchas de las acciones del personal de salud determinan la calidad de atención que reciben las mujeres durante el embarazo, parto y puerperio, acciones que a su vez repercuten en la salud individual de cada mujer que pisa una institución de salud, tanto a nivel físico como mental.

La culminación del proceso gestacional, es decir, el nacimiento, tiene muchas implicaciones para la mujer y el niño que esta por nacer. Éste es doloroso y existen casos en que el proceso de parto es un fenómeno de gran impacto para la mujer, mismos que se ven influenciados por la atención brindada por el personal de salud (1), es decir, si el trato que recibe la mujer no es digno, si su integridad física y mental no es protegida o de alguna manera se atenta contra ellas, se habla de la violación a sus derechos, no sólo como mujer, sino como ser humano, y esto se considera violencia.

En cuestión de género existen diferentes tipos de violencia contra la mujer, y de alguna forma se tipifica por el entorno en que se ejerce. Dentro de este referente social, existe un tipo de violencia contra la mujer embarazada que es ejercida dentro de las instituciones de salud por parte del personal que presta dichos servicios, ya sea en la atención al parto, cesárea o aborto, misma que tiene implicaciones legales serias, y es de denominada violencia obstétrica (2). A su vez, este tipo de violencia se expresa principalmente a través del llamado "trato deshumanizado" a la mujer embarazada, la medicalización de los procesos reproductivos naturales y todas aquellas expresiones que pudieran poner en riesgo a la mujer durante la atención a su salud sexual, embarazo, parto y post parto (3).

Si bien, la mujer puede ser víctima de violencia durante el embarazo, resulta de gran importancia aclarar que no será considerada como violencia obstétrica aquella que no haya sido ejercida por algún miembro del equipo de salud durante la atención de la mujer en estado de gravidez ${ }^{(2)}$. La violencia obstétrica es una forma específica de violación de los derechos humanos y reproductivos de la mujer, entre las cuales se incluyen aquellos actos que atenten contra sus derechos a la igualdad, no discriminación, información, integridad, salud y autonomía reproductiva ${ }^{(4)}$.

La también denominada violencia institucional, es considerada un factor de riesgo importante para que se produzca una muerte materna, sobre todo en aquellas mujeres que pertenecen a grupos vulnerables, como grupos indígenas y aquellos que viven en niveles extremos de pobreza ${ }^{(5)}$. El parto es considerado un proceso fisiológico que no debería implicar un riesgo para la vida de una mujer, por lo que este no debería requerir de múltiples intervenciones, sin embargo, a lo largo de los años su atención se ha vuelto un acto médico rutinario ${ }^{(2)}$ que implica diversas acciones que podrían, en determinado momento, poner en riesgo la integridad física y mental de las mujeres embarazadas.

En este contexto, la Organización Mundial de la Salud (OMS) publicó una serie de lineamientos referente a las prácticas inadecuadas utilizadas frecuentemente durante la atención al parto, entre las que destacan no hacer intervenciones médicas innecesarias, tales como la episiotomía, rasurado y enema como prácticas de rutina; restringir el uso de oxitocina, analgesia y anestesia (6).

Si bien, la violencia obstétrica es un problema de salud pública ampliamente conocido (7), son pocos los países que cuentan con una legislación que respalde a la mujer en relación a esto, entre los cuales se encuentra Venezuela, al ser el primero en América Latina en contar con una Ley sobre el derecho a las mujeres a una vida libre de violencia, que defina las conductas de rutina obstétrica que son considerados como violencia de género en los hospitales ${ }^{(8)}$. Si bien, en México no existe actualmente una ley que rija a nivel nacional lo referente a violencia obstétrica, se ha realizado diversos estudios que buscan definir la presencia, causas y percepción de las pacientes respecto a esto.

En el año 2015, en San Andrés Cholula, Puebla, se realizó una investigación de tipo transversal en 29 médicos que se desempeñan en el ejercicio de la obstetricia y ginecología, esta misma asociada con el Síndrome de Burnout (o desgaste profesional), obteniendo: negligencia médica y prácticas nocivas (35.71\%) y discriminación $(35.71 \%)^{(4)}$.

En el año 2015 en Caracas, Venezuela se realizó un estudio prospectivo y descriptivo, en el que se investigó la posibilidad de ocurrir violencia obstétrica en 326 pacientes, atendidas por partos cesáreas y abortos, como resultado la prevalencia de violencia fue de $26.3 \%$ (violencia psicológica) como detonante de los resultados. ${ }^{(2)}$ En el año 2013, en Caracas Venezuela, se realizó otro estudio sobre aspectos relativos a violencia obstétrica, el $66.8 \%$ manifestó la realización de procedimientos médicos sin consentimiento informado y un $49.4 \%$ fue objeto de algún tipo de trato deshumanizante ${ }^{(9)}$. Por otra parte, en el año 2012, en Cuernavaca Morelos, se realizó una investigación a mujeres en el posparto inmediato de 2 hospitales con el objetivo de evidenciar el abuso de las mujeres en las salas de parto. $11 \%$ de las mujeres refirió haberse sentido maltratadas por el personal de salud ${ }^{(7)}$.

En el noroeste de México no se ha localizado información sobre el tema en cuestión, ya que es una realidad que este tipo de violencia se ve como algo normal por parte de las usuarias del servicio de salud. En la sociedad, los daños son magnificados, debido a la escasa información 
de la misma temática, es por esto que surgió el interés por realizar una investigación que tuviera como objetivo conocer la percepción que tienen las usuarias atendidas en las unidades hospitalarias, públicas y privadas, sobre violencia obstétrica.

\section{METODOLOGÍA}

El diseño de la investigación fue cuantitativo, descriptiva y de cohorte transversal, ya que se midieron las variables y no se manipularon y se realizó la medición de las mismas en un sólo momento.

La población des estudio incluía a mujeres que hubieran tenido una o más gestas y que hubieran sido atendidas en una institución hospitalaria durante el parto, y excluía a todas aquellas que no hubieran tenido un embarazo completo. El tipo de muestreo fue no probabilístico por conveniencia a través de la técnica de bola de nieve, esta es una técnica de consiste en que las mujeres seleccionadas para ser estudiadas incorporan a otras mujeres que cumplan con los criterios entre sus conocidas, esto permitió que el tamaño de la muestra creciera a medida que las personas seleccionadas invitaron a sus conocidas. Por lo que la muestra final estuvo conformada por 45 participantes.

Los datos se recolectaron en el domicilio particular de las mujeres participantes, mediante el "Test para denunciar la violencia obstétrica en los hospitales extremeños" (TDVO) que consta de 12 preguntas, de las cuales 11 de ellas son dicotómicas con respuesta sí o no y 1 de elección múltiple. Dicho instrumento, en el presente estudio tuvo una fiabilidad por Alfa de Cronbach de .829.

Para la captura y procesamiento de los datos se utilizó el paquete estadístico SPSS para Windows versión 16. Para el análisis de los datos fue empleada estadística descriptiva, mediante frecuencias y proporciones y medidas de tendencias central.

Para el presente estudio se tomó en cuenta el reglamento de la ley general de salud en materia de investigación para la salud, de la cual se utilizó el titulo primero del cual se tomó en cuenta el artículo 3ro que nos habla acerca del conocimiento de los procesos biológicos y psicológicos de los seres humanos, el conocimiento entre causas de enfermedad y el estudio de técnicas y métodos que se emplean para la prestación de servicios. También fue considerado el titulo segundo de los aspectos éticos de la investigación en seres humanos en el capítulo, del cual se utilizan varios artículos como el artículo 13, que nos habla de respeto a la dignidad, protección de los derechos de la persona y su bienestar, el artículo 14 que nos habla acerca de las bases fundamentales que se deben llevar a cabo en las investigaciones con seres humanos, el artículo 16 que nos habla acerca de la privacidad del individuo investigado, el artículo 21 explicarle al sujeto los objetivos de la investigación, que procedimientos se llevara a cabo, los beneficios que se pueden observarse u obtener, por último el articulo 22 nos habla acerca de los requisitos que se deben de reunir para poder contar con el consentimiento informado ${ }^{(10)}$.

\section{RESULTADOS}

En referencia a los datos sociodemográficos, del total de la muestra estudiada $(n=45)$ el $75.6 \%$ de las mujeres pertenece al grupo etario de 18 a 25 años, con una edad media de 22.53 años, una $D E=3.88$, con una mínima de 16 y máxima de 32 años. Respecto al estado civil la mayoría de las mujeres son solteras (46.2\%), siguiéndole aquellas casadas (42.2\%); en cuanto a la situación laboral actual el $55.6 \%$ se encuentra desempleada y el $88.9 \%$ practican la religión católica. A su vez, el $68.9 \%$ de las participantes proceden de Hermosillo y en cuanto al tipo de institución en donde se atienden el 82.2\% lo hace en institución pública (Tabla 1).

\section{TABLA 1. DATOS SOCIODEMOGRAFICOS}

\begin{tabular}{|l|r|r|}
\hline Variables & $f r$ & $\%$ \\
\hline Edad & 3 & \\
Menores de 18 años & 34 & 75.6 \\
18 a 25 años & 8 & 17.8 \\
25 a 35 años & & \\
\hline Estado civil & 21 & 46.7 \\
Soltero & 19 & 42.2 \\
Casado & 4 & 8.9 \\
Unión libre & 1 & 2.2 \\
Divorciada & & \\
\hline Situación laboral & 20 & 44.4 \\
Empleado & 25 & 55.6 \\
Desempleado & & \\
\hline Religión & 40 & 88.9 \\
Católica & 4 & 8.9 \\
Cristiana & 1 & 2.2 \\
Ateo & & \\
\hline Lugar de procedencia & 31 & 68.9 \\
Hermosillo & 14 & 31.1 \\
\hline Otro & & \\
\hline Tipo de institución donde se atiende & 37 & 82.2 \\
Pública & & 17.8 \\
\hline Privada & & 100 \\
\hline TOTAL & & \\
\hline \multicolumn{1}{|c|}{ Fuente: } & & \\
\hline
\end{tabular}


En relación a los antecedentes gineco-obstétricos, más de la mitad de la muestra (51.1\%) dicen haber tenido una gesta, a su vez $57.8 \%$ dice haber tenido 1 parto, $31.1 \% 1$ cesárea y $84.4 \%$ dice no haber tenido abortos a lo largo de su vida (Tabla 2). De igual forma, se obtuvo una media de gestaciones de 1.80, con una desviación $D E=1.014$, una mínima de 1 y máxima de 5 gestaciones.

TABLA 2. ANTECEDENTES GINECO-OBSTETRICOS

\begin{tabular}{|c|c|c|}
\hline Variables & $f r$ & $\%$ \\
\hline \multicolumn{3}{|l|}{ Gestaciones } \\
\hline 1 & 23 & 51.1 \\
\hline 2 & 12 & 26.7 \\
\hline 3 & 7 & 15.6 \\
\hline 4 & 2 & 4.4 \\
\hline 5 & 1 & 2.2 \\
\hline \multicolumn{3}{|l|}{ Partos } \\
\hline 0 & 7 & 15.6 \\
\hline 1 & 26 & 57.8 \\
\hline 2 & 9 & 20.0 \\
\hline 3 & 3 & 6.7 \\
\hline \multicolumn{3}{|l|}{ Cesárea } \\
\hline 0 & 29 & 64.4 \\
\hline 1 & 14 & 31.1 \\
\hline 2 & 2 & 4.4 \\
\hline \multicolumn{3}{|l|}{ Abortos } \\
\hline 0 & 38 & 84.4 \\
\hline 1 & 4 & 8.9 \\
\hline 2 & 3 & 6.7 \\
\hline TOTAL & 45 & 100 \\
\hline
\end{tabular}

Por otra parte, respecto a los resultados obtenidos en el test sobre violencia obstétrica, $77.8 \%$ de las usuarias dice no haber sido criticada por su comportamiento con comentarios irónicos, descalificadores o en tono de chiste. El $71.1 \%$ respondió que no al cuestionamiento "¿La trataron con sobrenombres o diminutivos o modificando su nombre en tono cariñoso como si fuera una niña incapaz de comprender las intervenciones que están realizando o el proceso de parto?". Sin embargo, el $44.4 \%$ dijo haber sido criticada por llorar o gritar de dolor, emoción, alegría o tristeza durante el trabajo de parto, 37.8\% afirma que le resultó difícil o imposible aclarar sus dudas y manifestar sus medios o inquietudes porque no le respondían o lo hacían de mala manera. A su vez, con respecto a los procedimientos que se le realizaron a las usuarias sin pedirles consentimiento o informarle sobre los riesgos y sin explicar por qué eran necesarios, los más frecuentes fueron episiotomía (46.7\%), prohibición de ingerir alimentos o bebidas (42.2\%), tactos vaginales reiterados y por diferentes personas (40.0\%) y administración de medicación para acelerar el parto (31.1\%). De igual forma, en relación al trabajo de parto el $35.6 \%$ de las mujeres dice que se le obligó a permanecer acostada boca arriba, aunque refiriera incomodidad, $51.1 \%$ dice que la privaron de la posibilidad de caminar o buscar posiciones seguras y $71.1 \%$ indica que se le impidió estar acompañada por alguien de confianza durante el proceso. En relación a la etapa de expulsión $48.9 \%$ de las usuarias dice que le impidieron tomar la postura más cómoda para pujar. Resalta que $73.3 \%$ dice que no se le impidió el contacto inmediato con su hijo/a, 75.6\% niega que la experiencia en la atención del parto la hubiera hecho sentir vulnerable, incapaz, culpable o insegura. Por otro lado, $84.4 \%$ desconoce alguna ley que la respalde contra la violencia obstétrica (Tabla 3).

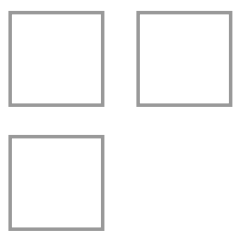


TABLA 3. RESULTADO DEL TEST DE PERCEPCIÓN DE VIOLENCIA OBSTÉTRICA.

\begin{tabular}{|c|c|c|c|c|}
\hline \multirow[t]{2}{*}{ Variables } & \multicolumn{2}{|c|}{ SI } & \multicolumn{2}{|c|}{$\mathrm{NO}$} \\
\hline & fr & $\%$ & $f r$ & $\%$ \\
\hline $\begin{array}{l}\text { 1. ¿El personal de salud criticaba tu comportamiento con comentarios irónicos, descalifica- } \\
\text { dores o en tono de chiste? }\end{array}$ & 10 & 22.2 & 35 & 77.8 \\
\hline $\begin{array}{l}\text { 2. ¿La trataron con sobrenombres o diminutivos o modificando tu nombre en tono cariñoso } \\
\text { como si fuera una niña incapaz de comprender las intervenciones que están realizando o el } \\
\text { proceso de parto? }\end{array}$ & 13 & 28.9 & 32 & 71.1 \\
\hline $\begin{array}{l}\text { 3. ¿Fue criticada por llorar o gritar de dolor, emoción, alegría o tristeza durante el trabajo } \\
\text { de parto? }\end{array}$ & 20 & 44.4 & 25 & 55.6 \\
\hline $\begin{array}{l}\text { 4. ¿Le resulto difícil o imposible aclarar sus dudas y manifestar sus medios o inquietudes } \\
\text { porque no le repondrían o lo hacían de mala manera? }\end{array}$ & 17 & 37.8 & 28 & 62.2 \\
\hline $\begin{array}{l}\text { 5. ¿Se le realizaron alguno o varios de los siguientes procedimientos sin pedirle consenti- } \\
\text { miento o informarle de los riesgos sin explicarle porque eran necesarios?, señalar cuales: } \\
\text { - Prohibición de ingerir alimentos o bebidas } \\
\text { - Indicación de permanecer acostada todo el tiempo } \\
\text { - Enema } \\
\text { - Rotura artificial de la bolsa } \\
\text { - Rasurado de genitales } \\
\text { - Administración de medicación para acelerar el parto } \\
\text { - Tactos vaginales reiterados y por diferentes personas } \\
\text { - Comprensión del abdomen en el momento del parto } \\
\text { - Episiotomía } \\
\text { - Cesárea } \\
\text { - Legrado }\end{array}$ & $\begin{array}{r}4 \\
6 \\
14 \\
18 \\
8 \\
21 \\
6\end{array}$ & $\begin{array}{r}8.9 \\
13.3 \\
\mathbf{3 1 . 1} \\
\mathbf{4 0 . 0} \\
17.8 \\
\mathbf{4 6 . 7} \\
13.3 \\
15.6\end{array}$ & $\begin{array}{l}41 \\
39 \\
31 \\
27 \\
37 \\
24 \\
39 \\
38\end{array}$ & $\begin{array}{l}57.8 \\
73.3 \\
82.2 \\
91.1 \\
86.7 \\
68.9 \\
60.0 \\
82.2 \\
53.3 \\
86.7 \\
84.4\end{array}$ \\
\hline $\begin{array}{l}\text { 6. ¿Durante el periodo de dilatación la obligaron a permanecer acostada boca arriba, aun- } \\
\text { que refiriera incomodidad? }\end{array}$ & 16 & 35.6 & 29 & 64.4 \\
\hline 7. ¿La privaron de la posibilidad de caminar o buscar posiciones según sus necesidades? & 23 & 51.1 & 22 & 48.9 \\
\hline $\begin{array}{l}\text { 8. ¿Durante el momento de expulsión le impidieron tomar la postura más cómoda para us- } \\
\text { ted para pujar? }\end{array}$ & 22 & 48.9 & 23 & 51.1 \\
\hline 9. Durante todo el proceso ¿Se le impidió estar acompañada por alguien de confianza? & 32 & 71.1 & 13 & 28.9 \\
\hline $\begin{array}{l}\text { 10. ¿Al momento del nacimiento se le impidió el contacto inmediato con su hijo/a, antes } \\
\text { de que se realizaran cuidados o intervenciones rutinarias (pesar, medir, administración de } \\
\text { vacunas y baño)? }\end{array}$ & 12 & 26.7 & 33 & 73.3 \\
\hline $\begin{array}{l}\text { 11. ¿La experiencia de la atención en el parto la hizo sentir vulnerable, incapaz, culpable o } \\
\text { insegura? }\end{array}$ & 11 & 24.4 & 34 & 75.6 \\
\hline 12. ¿Conoce alguna ley que la respalde contra la violencia obstétrica? & 7 & 15.6 & 38 & 84.4 \\
\hline
\end{tabular}

Fuente: TDVO $n=45$ 
Adicionalmente, el trato deshumanizante osciló mayormente en usuarias entre 16 y 21 años de edad, y disminuyó gradualmente en mujeres entre 22 y 27 años de edad. En el caso de mujeres que tienen entre 28 y 32 años de edad, la frecuencia fue mínima. Siendo las mujeres que tienen menor edad, por la probable inexperiencia del parto, consideren esta clase de actos como parte del proceso, ya que, al momento de aplicar algunas encuestas, varias de ellas manifestaron que no sabían que estaban siendo violentadas hasta que realizaron la encuesta.

Finalmente, al considerar el instrumento de manera globalizada, se sumaron los ítems obteniendo que, a mayor puntuación, al asignarles una puntuación positiva cuando la respuesta era afirmativa, la mujer percibía un mayor grado de violencia obstétrica. Obteniendo que la puntuación media fue de 15.16, con una mínima de 6 y máxima de 22. Por lo que se observa que la puntuación media refleja un grado de violencia que podría considerarse moderado.

\section{DISCUSIÓN}

Al contrastar los datos encontrados en este estudio con los encontrados en los realizados con anterioridad. En relación a los datos sociodemográficos en este estudio se encontró que la mayoría de las mujeres pertenecían al grupo etario de $18-25$ años (75.6\%), lo que concuerda con lo encontrado por Valdez-Santiago y otros ${ }^{(7)}$, donde el $64 \%$ de la muestra correspondía al grupo etario de 1324 años, a su vez con en este estudio se encontró una edad media de 22.53 años, lo que concuerda con lo encontrado también por Pereira y otros ${ }^{(2)}$, quienes obtuvieron una edad media de 22.5 años.

En este estudio la mayoría de las participantes son primigestas, lo que difiere con lo encontrado con en otro estudio donde más de la mitad de las mujeres fueron multigestas. En el mismo estudio también se encontró diferencia en relación al número de embarazos, donde la media fue de 3 , mientras que en este estudio fue de 1.8 embarazos ${ }^{(7)}$.

En relación al cuestionario, en este estudio, la mayoría de las mujeres dijeron que no se les permitió estar acompañada durante el proceso (71.1\%), sin embargo, en los estudios relacionados no se encontró evidencia de que este aspecto haya sido evaluado como acto de violencia obstétrica. A su vez, en este estudio se encontró que la afirmación de segunda mayor incidencia fue aquella en que las mujeres dijeron que no se les permitió caminar o buscar la posición más cómoda (51.1\%), lo que en porcentaje es mayor a lo encontrado en otro estudio donde este hallazgo tuvo un porcentaje afirmativo del $24.9 \%{ }^{(9)}$.

Dentro de los estudios relacionados en comparación con este, el hallazgo común de mayor prevalencia es que la mayoría, o gran parte de las mujeres, dijeron haber sido criticadas por llorar o gritar de dolor, emoción, alegría o tristeza, práctica de la que han sido víctimas muchas mujeres en diferencias períodos y entornos (7) (2) (9).

Finalmente, en relación a los procedimientos realizados sin consentimiento o sin haberle explicado, en este estudio el de mayor frecuencia fue la episiotomía (46.7\%), lo que difiere con otros estudios realizados, donde el de mayor incidencia fue la realización del tacto vaginal ${ }^{(9)(7)(3)}$.

\section{CONCLUSIONES}

La violencia obstétrica, se encuentra definida en el artículo 3, apartado IX de la Ley de Acceso de las Mujeres a una Vida Libre de Violencia del Estado de San Luis Potosí, como también todas aquellas actividades que se consideren abuso, conducta, acción u omisión intencional, negligente y dolorosa contra la mujer durante el embarazo, parto o puerperio ${ }^{(11)}$. En la presente investigación se demostró, con base a los resultados que, la violencia obstétrica es una realidad dentro de las instituciones de salud en México, y el estado de Sonora no es la excepción.

Si bien, en la presente investigación los porcentajes mayores no se inclinan por lo afirmativo en cuestión de acciones consideradas como actos de violencia obstétrica hacia las mujeres embarazadas, es de gran relevancia destacar que dichos actos están presentes en porcentajes considerables, según lo expresado por las mujeres sonorenses que participaron.

Con estos resultados, se muestra que a pesar de los años transcurridos y de muchas acciones implementadas, las mujeres embarazadas son víctimas de violencia obstétrica dentro de las instituciones, pero sin percibirla como tal hasta el momento de cuestionarle y aclararle cuáles son las manifestaciones de dicha violencia. La mujer es víctima de distintos tipos de violencia, aún cuando se cuenta con avances tecnológicos e investigaciones, la práctica del cuidado de la mujer parturienta se ha ido mecanizando y desensibilizando, generando con esto experiencias de partos traumáticos.

En México no todos los estados cuentan con leyes que protejan contra la violencia obstétrica. En algunos estados del país hay artículos que definen la violencia obstétrica como son San Luis Potosí, Chihuahua, Durango, Guanajuato, Tamaulipas, Veracruz, Quintana Roo y Chiapas, son estados que ya tienen un avance acerca de las leyes contra la violencia obstétrica. Actualmente se encuentran pendientes de dictaminar en el Congreso de la Unión seis iniciativas para definir la violencia obstétrica en la Ley general de acceso de las mujeres a una vida libre de violencia. ${ }^{(11) .}$

Es importante destacar de esta investigación la inexistencia de alguna ley que proteja a la mujer contra la violencia obstétrica en Sonora, como sucede en otros estados 
de la república y la falta de conocimiento de algunas usuarias sobre el trabajo de parto.

Debido a esto, y en base a las recomendaciones establecidas por los organismos a nivel mundial en México se ha visto la necesidad de establecer propuestas para la atención materna y perinatal, donde la atención se brinde bajo un modelo con perspectiva de género e interculturalidad como parte del marco normativo que rige los servicios de salud ${ }^{(12)}$.

Finalmente, como personal de salud, las enfermeras $y$ enfermeros tienen que hacerse participes en la im- plementación de buenas prácticas en la atención a la mujer embarazada, no sólo en la atención directa en las salas de parto, ya que también es obligación del profesional de enfermería abogar por los derechos de las usuarias en esta etapa tan importante de sus vidas. Esto se puede lograr a través de la educación de las usuarias y el personal de salud en materia de derecho, donde el fin común es dar y recibir una atención de calidad, humanizada y profesional que proteja la integridad física y mental de la mujer y su hijo, que a largo plazo, tenga un impacto positivo sobre las tasas de mortalidad materno-infantil en nuestro país.

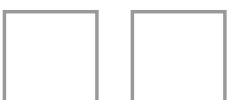




\section{REFERENCIAS BIBLIOGRÁFICAS}

1. Aguirre A, Martínez B, Rivero K, Blancas C, Badillo S, González L, et al. La atención del parto: poder, derechos, violencia. Revista Salud Quintana Roo. 2013; 6(25): p. 21-5.

2. Pereira C, Domínguez A, Toro J. Violencia Obstetrica desde la perspectiva de la paciente. Revista de Obstetricia y Ginecologia de Venezuela. 2015; 75(2): p. 81-90.

3. Soto-Toussaint L. Violencia Obstétrica. Revista Mexicana de Anestesiología. 2016 Abril-Junio; 39(S1): p. S55-S60.

4. Pintado-Cucarella S. Síndrome de desgaste profesional en médicos y percepciónde la violencias obstétrica. Gineco Obstet Méx. 2015; 83: p. 173-8.

5. Tinoco MT. Propuesta para la prevención y atención de violencia institucional en la atención obstétrica en México. Revista CONAMED. 2016; 21(1): p. 48-54.

6. Organización Mundial de la Salud (OMS). Declaración de la OMS sobre tasas de cesárea. [Online].; 2015 [cited 2016 Mayo 06. Available from: http://www.who.int/reproductivehealth/publications/maternal_perinatal_health/cs-statement/es/.

7. Valdez-Santiago R, E HS, Mojarro-Iñiguez M, Arenas-Monreal L. Nueva evidencia a un viejo problema: el abuso de las mujeres en las salas de parto. REvista CONAMED. 2013; 18(1): p. 14-20.

8. Camacaro M, Ramírez M, Lanza L, Herrera M. Conductas de rutina en la atención al parto constitutivas de violencia obstétrica. Notas y debates de actualidad. 2015; 20(68): p. 113-20.

9. Teran P, Castellanos C, González M, Ramos D. Violencia obstetrica: percepción de las usuarias. Revista Venezolana Gineco-obstetrica. 2013; 73(3): p. 171-80.

10. [DOF], Diario Oficial de la Federación. Ley General de Salud: Reglamento de la Ley General de Salud en Materia de Investigación para la Salud. México:; 1984.

11. Grupo de Información en Reproducción Elegida [GIRE]. Violencia obstétrica en leyes de acceso a una vida libre de violencia. [Online].; 2015 [cited 2016 Agosto 15. Available from: https://gire.org.mx/consultations/leyes-locales-de-acceso-a-una-vida-libre-de-violencia/?type $=$.

12. Villanueva L, Ahuja M, Valdez R, Lezana M. ¿De qué hablamos cuando hablamos de violencia obstétrica? Revista CONAMED. 2016; 21(S1): p. 7-25.

13. Organización Mundial de la Salud (OMS). Episiotomía en el parto vaginal. [Online].; 2008 [cited 2016 Diciembre. Available from: https:/extranet.who.int/rhl/es/topics/ pregnancy-and-childbirth/care-during-childbirth/care-during-labour-2nd-stage-6. 